to explain the lattice dynamics of simple ionic crystals. Recently Stirling using a similar model has satisfactorily fitted the experimental measurements for $\mathrm{SrTlO}_{3}$. The theory has been extended by Cowley and Bruce to include anharmonic effects. This is the area which is currently of greatest interest to those concerned with structural phase transitions, and which must eventually succeed in connecting the lattice dynamics approach to the more orthodox approaches to the theory of phase transitions.

The theory of a new phenomenon in acoustics called "phonon echoes" was given by the very interesting paper of J. Joffrin, Université Paris VI. Laboratoire d'UItrasons, in terms of a classical presentation. The essential properties which are the time reversal, the node to node coupling, and the existence of a non-linear interaction of phonons with an external electric field were described. The applications for the physicist and for the engineer were also briefly reported.

K. Dransfeld, Max-Planck-Institut für Festkörperforschung HochfeldMagnetlabor Grenoble, summarized in his talk the new results of the dynamical properties of disordered materials at very low temperatures (below $\left.1^{\circ} \mathrm{K}\right)$. All these observations are consistent with a new type of low lying amorphous materials but which are absent in perfect crystals.

Both light and neutron scattering methods are extensively used to study phonon spectra of solids. A comparison between the two methods was made by N. Kroó, Central Research Institute for Physics, Budapest, in order to show the similarities and differences, with typical examples given mainly in those fields where the applied method is superior.
The physics and chemistry of solid surfaces exposed to vacuum or to gases are proceeding very fast. G. Heiland's (2. Physikalisches Institut der Rheinisch-Westfälischen Technischen Hochschule, Aachen) valuable and detailed report on the new results in this field was therefore of topical interest. Reliable methods for the preparation of clean surfaces and new ways of characterization and investigation provide meaningful data on a much wider scale than a few years ago. Stimulated by recent experimental results, the theoretical activity has grown considerably. Driving forces for research on surfaces originate not only from theory, but mainly from applications in solid state electronics and in heterogeneous catalysis. New experimental methods of surface research are the following:

(i) the surface crystallographic structure and its transitions can be recognized by the elastic scattering of low energy electrons and the atomic steps analysed;

(ii) inelastic scattering of electrons, photoemission, photoconductivity and ellipsometry have been used for the spectroscopy of vibronic and electronic surface states;

(iii) new data on interactions between solid surfaces and molecules is contributed by molecular beam mass spectroscopy.

From written and oral comments, the Organising Committee of the Conference had the distinct feeling that the conference had been warmly received, and that participants from both subject groups had benefited from this contact with each other.

G. Szigeti, Budapest

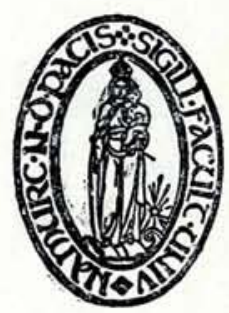

The Physics Department of the Facultés Universitaires de $\mathrm{Na}$ mur (Belgium) invites applications for a permanent position at the

\section{Chargé de Cours}

level, from French speaking $\mathrm{Ph}$. D.'s with exceptional qualifications in both teaching and research.

Teaching duties will consist in general physics courses in candidatures in sciences. Research responsibilities will include experimental or/and theoretical research in the following existing interest groups: atomic, molecular and electronic spectroscopies ; theoretical and mathematical physics ; solid state physics; applied low energy nuclear physics.

Applications forms may be requested from the

Service du Personnel

des Facultés Universitaires

Notre-Dame de la Paix,

61 , rue de Bruxelles,

B-5000 Namur.

\title{
1st EPS Conference of the Nuclear Physics Division on Nuclear Interactions at Medium and Low Energies
}

\section{Harwell, England, 24-26 March 1975}

The Nuclear Physics Division of the EPS is at present cooperating with National Societies to create a series of European topical conferences in the field of nuclear physics. Two conferences per year are planned.

The first of this series, organized in cooperation with the Institute of Physics, was held at the UKAEA Harwell Laboratory in Britain on 24-26 March 1975 on the topic of nuclear interactions at medium and low energies'. The conference attracted about 170 participants, more than a third of whom came from continental Europe.

In his welcoming speech B. Rose (Harwell) was guardedly optimistic about the future of nuclear structure physics. For British physicists the new and upgraded machines at Daresbury and Oxford and the use of the highflux neutron facility at the three-nation Laue-Langevin Institute in Grenoble were welcome developments. The main problem, as elsewhere in Europe, seemed to be the maintenance of a steady input of good people into the field.

The subject that produced the most interesting contributions and stimulated the most discussion was that of heavy ion physics, one of the growth areas of recent years. The application of heavy ion reactions to nuclear spectroscopy both extends and complements the information obtainable from light ion reactions. The first invited talk in the opening session, which was chaired by the Chairman

1) The heavy ion conference held three years earlier at Aix-en-Provence, France, should probably be termed the zeroth divisional conference, since the Nuclear Physics Division had not then been officially formed. 
of the N.P. Division of the EPS, was devoted to this subject and was delivered by W.R. Phillips (Manchester). The high charge and angular momentum introduced in a heavy ion reaction lead respectively to appreciable cross sections for multiple Coulomb excitation (even to states as high as $18^{+}$) and to the population of high spin states in heavy and light compound reactions. So many reaction channels are open in the latter type of reaction that things are still rather at the "bird-watching" stage. The high linear momentum brought in a heavy ion reaction which leads to high recoil velocities of the reaction products, and enables the use of the recoildistance method of determining lifetimes and magnetic moments of excited states.

The determination of dipole moments makes use of the hyperfine interaction in highly stripped ions. A measurement on the Manchester $\mathrm{HI}$ LAC attempting to use the magnetic field from $L$-shell vacancies following scattering of $150 \mathrm{MeV}{ }^{56} \mathrm{Fe}$ was described. One $\gamma$-ray spectrum taken in three hours could be obtained in one minute on a $15 \mathrm{MV}$ tandem accelerator. Heavy ion transfer reactions including cluster transfers and two-step processes were dealt with both here and in contributions to the parallel sessions by, for example, groups from Oxford and Heidelberg. A more exotic measurement, in which a search was made for heavy-ion-produced $\pi^{\circ}$ mesons, was also reported.

A.M. Lefort (Orsay) widened the discussion to include hard grazing collisions between heavy ions and quasifission. He illustrated the kind of information that can be obtained by measuring the angular and mass distributions of the fragments produced with a wide variety of projectiles and targets over a wide range of bombarding energy and also discussed the role of classical concepts like friction in the reaction process. In particular types of collision up to $200 \mathrm{~h}$ of angular momentum may be

The Nuclear Physics Division of EPS currently counts 286 members but would welcome new applications.

The members of the Board are listed below:

C. van der Leun, Utrecht, (Chairman); A. Strzalkowski, Cracow, (Vice-Chairman); W.D. Hamilton, Brighton, (Secretary); K. Alder, Basel; K. Bethge, Heidelberg; N. Cindro, Zagreb; E. Cotton, Git-surYvette; P.G. Hansen, Geneva; M. Ivascu, Bucharest; T. Mayer-Kuckuk, Bonn; S.G. Nilsson, Lund; R.A. Ricci, Legnaro; I. Talmi, Rehovot.

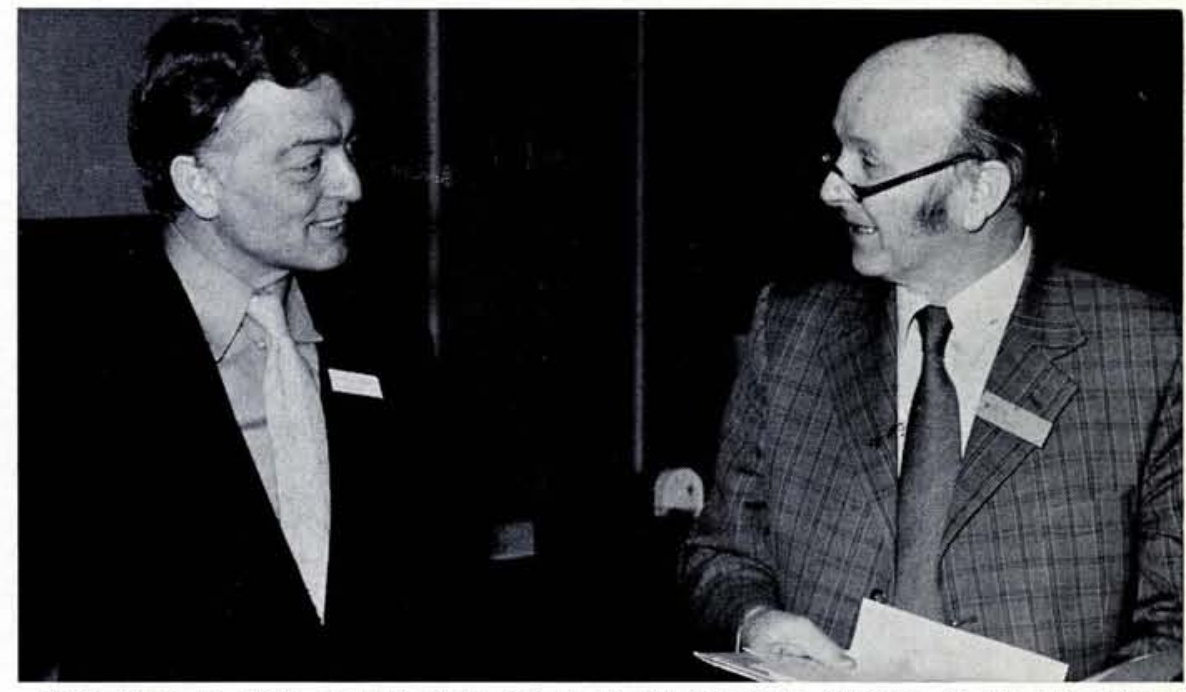

Dr B. Rose, of AERE, Harwell, right, and Dr C. Van der Leun, Chairman of the N.P. Division at the Harwell Conference.

introduced and the resulting rotational energy of the system is very large.

The application of heavy ion reactions to the neighbouring field of atomic physics, and in particular to problems in quantum electrodynamics, was treated very clearly by W. Greiner (Frankfurt), who started from first principles in discussing the properties of super-heavy atoms created fleetingly in heavy ion collisions. At high enough atomic number the 1s states of the united atoms are expected to dive into the negative-energy continuum with the creation of electronpositron pairs. In some cases molecular X-rays from the K-, L- and $\mathrm{M}$ shells of colliding atoms have already been observed, but until now below the critical atomic number. A new level of sophistication will have been reached, when coincidence experiments become possible.

Fission, the second main subject at the conference, was dealt with both by H.J. Specht (Heidelberg) and in contributed papers, which included such topics as electron-induced fission. Specht summarized present knowledge and posed a number of questions that could only be answered by further experimental investigation, such as whether the nucleus has a permanent octupole shape at the second barrier, whether the shape at the final saddle points influences the mass distribution and whether there are different barrier heights for symmetric and asymmetric fission.

The solid work being done in lowenergy neutron interactions to test the statistical behaviour of the compound nucleus and an evaluation of the standard methods of detection and measurement of neutrons were dealt with by $\mathrm{H}$. Weigmann (Geel) and M.S. Coates (Harwell), respectively. M. Ashgar (Grenoble) described the new Lohengrin mass spectrometer at the Laue-Langevin Institute. This is a parabola-type machine, in which the perpendicular electric and magnetic fields are separated in space and which is beginning to produce some unique results.

Beta- and gamma-ray spectroscopy is an example of the kind of subject which falls within the budget of the smaller nuclear laboratories, and a separate parallel session was devoted to it.

Fields bordering on nuclear interactions at medium and low energy were given rather more prominence than might have been expected in a three-day conference, but the talks (perhaps for this reason) were rather entertaining. D. Bodansky (Seattle) provided the cosmological background to the production and abundance of the light elements that every experimentalist measuring proton- or alpha-bombardment or neutron-capture cross sections could wish to know. J.P. Blaser (Zürich) reported on the present status of the meson factory SIN, from which it is hoped to produce $1 \mathrm{~mA}$ of $\pi$ and $\mu$ mesons at $500 \mathrm{MeV}$, mainly to be used for high energy physics research, but also for medical purposes. An enthousiastic reception was given to D. Ashby (Harwell), who introduced a science-fiction-like note into the proceedings with his discussion of the futuristic laser-fusion technology at present being developed in different parts of the world in the hope of providing abundant, clean energy.

Various extra-curricular activities enlivened what little time was left over from confering. These included visits to nearby laboratories, sherry parties in the Oxford colleges in which the participants were lodged, and a concert of chamber music in the oldest music room in Britain built for public recitals.

H.A. Doubt, Utrecht 